\title{
Danza en educación infantil: opinión de los docentes Dance in preschool education: teachers' opinion
}

Vicente Juan Peña de Hornos, Gregorio Vicente Nicolás

Universidad de Murcia (España)

\begin{abstract}
Resumen. El objetivo principal de esta investigación ha sido conocer y analizar la inclusión de actividades de danza en la práctica educativa del aula de Educación Infantil desde la perspectiva de los docentes. Los participantes ( $\mathrm{N}=105)$ han sido maestras/os que imparten docencia en el segundo ciclo de Educación Infantil en centros educativos de la Región de Murcia (España). Para la recogida de datos se ha diseñado un cuestionario ad hoc y posteriormente se ha aplicado un análisis descriptivo a la información obtenida. Los resultados reflejan que los especialistas de Educación Infantil de la Región de Murcia incluyen en sus programaciones y en su práctica docente actividades de danza. Asimismo, las consideran fundamentales en el desarrollo integral del alumnado y que este responde de forma positiva y activa a este tipo de actividades. Por otro lado, alertan de la necesidad de mejorar la oferta formativa con respecto a estas materias y la calidad y cantidad de medios y recursos para llevarlas adecuadamente a la práctica.
\end{abstract}

Palabras clave: danza, expresión corporal, formación del profesorado, Educación Infantil, currículo.

\begin{abstract}
The main objective of this research was to learn and analyze the inclusion of dance activities in Preschool teaching practices from the perspective of teachers. Participants $(\mathrm{N}=105)$ were teachers who teach at the second cycle of Preschool Education in schools from the Region of Murcia (Spain). An ad hoc questionnaire was designed for the collection of data, and descriptive analysis was subsequently applied. The results reflect that specialists of Preschool Education from the Region of Murcia include dance activities in their planning and teaching practices. Likewise, they consider them fundamental for the integral development of students, who respond in a positive and active way to this type of activities. On the other hand, they call the attention on the need to improve both teacher training with respect to these subjects and the quality and quantity of resources to carry them properly into practice.
\end{abstract}

Key words: dancing, body language, teacher training, Preschool Education, educational syllabus.

\section{Introducción}

La Historia de la Educación ha ofrecido numerosos testimonios de la inclusión de la danza en la formación del ser humano y ha estado presente en las diversas manifestaciones en las que esta se ha llevado a cabo (Pedrero, 2013). Sin embargo, «el alcance y repercusión que tuvo en el ámbito educativo no fue muy generoso y prolífero» (Vicente, Ureña, Gómez, \& Carrillo, 2010, p.43). En el caso de España, aunque los avances han sido importantes, aún queda mucho camino por recorrer, debido a que la danza todavía no ha adquirido la categoría de materia en el ámbito de la educación formal obligatoria y únicamente forma parte de los contenidos de otras áreas como Artística, Música o Educación Física. A la anterior, habría que añadir otras razones mencionadas hace décadas por Paulson (1993, citado en citado en Vicente, et al., 2010) que esclarecen la limitada incorporación de la danza en la práctica educativa. Entre ellas destacan las siguientes: escasa formación del profesorado, inexistencia de un currículo especializado de danza, carestía de medios y material bibliográfico, insuficientes espacios adecuados o prevalencia de estereotipos sociales que la conciben desde una perspectiva de género discriminatoria.

Sin embargo, «la danza no es indiferente a la educación, es una herramienta que promueve la cultura y la formación de la personalidad y otras dimensiones» (Bernal, 2016, p.135). Por este motivo, Bernal asevera que la danza es esencial en la educación desde preescolar y primaria para el desarrollo de habilidades motrices, pero sobre todo comunicativas. Al res-

Fecha recepción: 11-01-19. Fecha de aceptación: 18-02-19 Gregorio Vicente Nicolás gvicente@um.es pecto, Ríos (2017, p.107) afirma que «para que la danza pueda considerarse en su dimensión educativa, debe favorecer el desarrollo integral del individuo». Ferreira (2009) va más allá, y considera que educar por medio de la danza es el punto de partida para desarrollar la creatividad, como evolución permanente para la vida.

Otros autores recuerdan la importancia de esta actividad en el desarrollo intelectual (Pérez-Aldeguer, 2012), o consideran que el elemento promotor de todo aprendizaje en el niño es el cuerpo (Fernández \& Arias, 2013). No es de extrañar que los niños en edad escolar tengan una necesidad imperiosa de movimiento, razón por la que diversos autores (Lení \& Wey, 2015; Martín, 2005; Pedrero, 2013; Ríos, 2017) enfatizan la necesidad de su educación desde edades tempranas.

Del mismo modo, el movimiento es básico en la construcción de la expresividad, en la capacidad reflexiva y en el reconocimiento de las posibilidades y los límites de los niños, pero estos también deben interactuar con otras personas para desarrollar la sociabilidad y la afectividad, interacción que sólo se produce a través de la comunicación. Este proceso se realiza por medio del pensamiento y se expresa a través del lenguaje y la motricidad. El niño, al conocer y dominar su cuerpo se relaciona mejor con lo que le rodea, por ello se le debe brindar de oportunidades de movimiento a lo largo de la vida, y así permitir que explore y desarrolle todo su potencial (Águila \& López, 2019; Lení \& Wey, 2015). Con respecto a la comunicación y dimensión social de la danza, deben señalarse los trabajos de Llorens (2016) y Llorens y Díaz (2018), quienes abogan por un concepto más amplio del concepto danza en las aulas de infantil, la danza integrada, formada siempre por grupos heterogéneos.

En relación con el objeto de estudio de esta investiga- 
ción, la danza en el ámbito de la Educación Infantil, es necesario recordar que el actual sistema educativo español contempla el contenido de esta disciplina en el ámbito de la enseñanza formal, aunque no adquiere la categoría de área o materia, ni en ningún momento se menciona el lenguaje de la danza. En este sentido, Díaz (2018, p.117), enfatiza que «se necesita una coherente integración del arte corporal dentro del currículo por parte de las autoridades, no solamente como mecanismo de aprendizaje musical [...], sino como parte fundamental del desarrollo integral y equilibrado del individuo». El Real Decreto 1630/2006, por el que se establecen las enseñanzas mínimas del segundo ciclo de Educación Infantil, dispone que el lenguaje artístico hace referencia tanto al plástico como al musical, encuadrado como bloque dentro del área Lenguajes: Comunicación y representación, y expone que este lenguaje posibilita el desarrollo de capacidades vinculadas con la expresión corporal, entre otras. De igual forma, en el área de Conocimiento de sí mismo y autonomía personal también se encuentran contenidos en relación a la expresión corporal, mediante un bloque específico que hace referencia al cuerpo y la propia imagen, y otro alusivo al juego y movimiento. El propio RD 1630/2006 resalta que las experiencias del alumnado con el entorno ayudarán a que conozcan global y parcialmente su cuerpo, sus posibilidades perceptivas y motrices, y puedan así identificar las sensaciones que experimentan, disfrutar con ellas y aprovechar las posibilidades expresivas del cuerpo para manifestarlas. Asimismo, el currículo establece como finalidad para la etapa de Educación Infantil la contribución al desarrollo físico, afectivo, social e intelectual de los niños. Paradójicamente, en los principios generales de la etapa no se alude a las enseñanzas artísticas ni a la expresión corporal o la danza, aunque sí se incluyen contenidos relativos a estos en los lenguajes artístico y corporal.

La presencia de la actividad corporal expresiva en el currículo de Educación Infantil debería tener un reflejo en las aulas y, como afirma Bernal (2016), es necesaria la modificación de las prácticas tradicionales para la búsqueda de innovaciones en el aula que utilicen en mayor medida el cuerpo, porque a través de él también se piensa. Para alcanzar dicha transformación el docente de Educación Infantil debe de poseer una serie de competencias. Como señala el RD 1630/ 2006, estos docentes deben contribuir a que los niños desarrollen capacidades que les permitan conocer su propio cuerpo y el de los otros, sus posibilidades de acción y aprender a respetar las diferencias, además de habilidades comunicativas en diferentes lenguajes y formas de expresión. Por este motivo, se considera muy importante que el profesorado posea una formación adecuada, lo que incluye recursos metodológicos en expresión corporal, para permitir que su alumnado tome consciencia de su propio cuerpo, pueda expresarse con él y observe sus capacidades (Herranz \& López, 2015).

Para autores como Cañal \& Cañal (2001), es fundamental favorecer que el alumnado experimente todas sus posibilidades de movimiento y considera que «la labor del maestro o maestra es como la del escultor, bajo cuya atenta y vigilante mirada de pedagogos, el cuerpo del niño o la niña en movimiento se convierte en un instrumento sensible, perceptivo y expresivo a la vez» (p.18). Esta tarea no es fácil de llevar a cabo y exige una gran preparación de todos los agentes implicados, pues «ser especialista en estas materias no se improvisa, ni tampoco es suficiente la buena voluntad» (Cañal \& Cañal, 2001, p.18).

Teniendo en cuenta que el docente de infantil debe poseer competencias relacionadas con la enseñanza de expresión corporal y danza, y que esta no se puede improvisar, es oportuno evocar las palabras de la profesora de pedagogía musical y danza Verena Maschat recogidas en una entrevista realizada por Carrascosa (2015). En ella, Maschat considera que la formación del profesorado, bien sea inicial o permanente, es el eje principal de la calidad de la enseñanza e insiste en que a partir de una base teórica sólida, debe aportar las técnicas y destrezas suficientes con la finalidad de ser un modelo ideal para el alumnado.

Al respecto, los estudios de Troya \& Cuéllar (2013) evidencian la escasa formación en danza del profesorado en las diferentes etapas educativas, donde las tres cuartas partes de los participantes manifestaron no haberla trabajado en ninguna etapa y un $90 \%$ constataba la ausencia total de experiencias relacionadas en Educación Infantil.

En la misma línea, Cuéllar \& Pestano (2013) afirman que la oferta de la asignatura de Expresión Corporal en la universidad no se adapta a las demandas del currículo educativo y la necesidad de formación de los titulados. En su estudio evidencian que más de la mitad de los créditos de asignaturas relacionadas con la expresión corporal en las universidades españolas se impartían de forma optativa, lo que evidencia que la materia no era una prioridad formativa en los futuros docentes de Educación Infantil. No debe relegarse al olvido que este vacío existente en formación del docente en expresión corporal y danza conlleva en muchas ocasiones una escasa práctica diaria en sus aulas y, por lo tanto, podría condicionar el desarrollo integral del niño en esta etapa educativa.

Tras el análisis de la literatura previa existente, es significativo mencionar la necesidad de realizar más investigaciones que aborden estudios sobre danza en la etapa de Educación Infantil.

A tenor de los argumentos expuestos anteriormente, surgen algunas cuestiones que son el punto de partida de esta investigación. Entre ellas se destacan las siguientes: ¿programan los docentes de Educación Infantil actividades de danza en sus aulas?; ¿ están formados en esta disciplina?; ¿ ¿la consideran importante para el desarrollo integral del niño?; ¿qué tipo de actividades de danza practican?; ¿disponen de medios y recursos adecuados para implementarlas?... Teniendo en cuenta los interrogantes planteados, el objetivo principal de esta investigación es conocer y analizar la inclusión de actividades de danza en la práctica educativa del aula de Educación Infantil desde la perspectiva del docente de dicha etapa. De este objetivo se derivan otros secundarios como son: (1) identificar el grado de puesta en práctica de actividades danza en el aula de Educación Infantil; (2) conocer la actitud del alumnado durante la praxis de dichas actividades desde el punto de vista del profesorado; (3) indagar sobre la formación de los docentes en esta disciplina; y (4) determinar su opinión acerca de la disponibilidad de medios y recursos para llevar a la práctica actividades de danza. 


\section{Metodología}

A tenor de la finalidad de esta investigación, se ha optado por un método cuantitativo de tipo descriptivo expuesto por Bisquerra (2009), con la pretensión de interpretar y analizar la situación actual sobre la práctica de la danza en la etapa educativa de Infantil. A tal fin, se ha elaborado un cuestionario destinado a docentes de dicha etapa.

\section{Participantes}

Los participantes de este estudio han sido maestros/as que impartían docencia en el segundo ciclo de Educación Infantil en centros educativos de la Región de Murcia (España). Para la configuración de la muestra se seleccionaron por conveniencia 16 colegios de esta región. Estos centros se eligieron según los siguientes criterios: (1) que estuvieran representadas las diferentes comarcas de la Región; (2) que la proporción de centros de titularidad pública fuese similar al porcentaje de centros públicos que existen en esta comunidad autónoma; y (3) que los centros estuvieran ubicados en contextos urbanos y rurales. El total de la muestra ascendió a 105 docentes de Educación Infantil (90.5\% mujeres). Con respecto a la titularidad de los centros educativos de los docentes que cumplimentaron el cuestionario, un 81.9\% tenían un carácter público, mientras que el resto era de titularidad concertada. Se subraya la nula participación de colegios privados.

\section{Instrumento}

Para la recogida de la información se diseñó un cuestionario ad hoc en el que se planteaban 25 afirmaciones estructuradas en cuatro bloques: práctica en el aula, formación del profesorado, actitud del alumnado y disponibilidad de medios y recursos. Los participantes debían elegir entre cuatro opciones posibles que expresaban el grado de acuerdo o desacuerdo. Asimismo, el cuestionario incluía una carta de presentación donde se formulaba el objetivo del mismo, el tratamiento de la información y se agradecía la participación. La relación de afirmaciones que componen el cuestionario se cargaron mediante la herramienta Formularios de Google, eligiendo para cada una de ellas una respuesta tipo Likert. La selección de dicha herramienta estuvo justificada por las grandes ventajas que reporta: gran alcance, gratuidad, sencillez y rapidez.

La validez de contenido del instrumento se determinó mediante un comité de expertos formado por cinco profesores/as (dos universitarios y tres de Educación Infantil) con una dilatada trayectoria profesional y un amplio conocimiento en el ámbito de la danza. En el proceso de validación, el comité valoró cada afirmación del cuestionario indicando su grado de pertinencia con respecto al objeto de estudio, además de su grado de precisión y de adecuación desde el punto de vista de su definición y formulación sintáctica, así como una valoración global del mismo. Se diseñó una escala de validación para el juicio de expertos, cuyos resultados mostraron que tanto la validez de las dimensiones como la validez de los ítems, obtenían un valor muy alto (3.9 sobre 4). Además, se calculó el valor W de Kendall (.67), que permitió comprobar que existía concordancia entre los jueces con una intensidad moderada (valor $W .51$ a .70) y, por lo tanto, que el instrumento cumplía el requisito de validez de contenido. Finalmente, se acometieron algunas modificaciones tras la valoración final del comité de expertos.

\section{Procedimiento}

Al término del proceso de creación, validación y modificación del cuestionario, se contactó con un miembro del equipo docente de Educación Infantil de varios centros educativos que respondían a los criterios establecidos. Posteriormente, se distribuyó telemáticamente un enlace web del cuestionario online a todo el profesorado de los centros que habían mostrado su compromiso a cumplimentarlo. Se envió a más de 150 profesores y profesoras y se estableció un plazo de 20 días para su realización. Durante este periodo de tiempo, dos centros de titularidad concertada/privada informaron de su posición contraria a la cumplimentación del cuestionario, alegando prohibiciones a la realización de cualquier tipo de cuestionario que no proviniera de sus servicios centrales. Finalizado el plazo de entrega se habían recibido 105 cuestionarios.

\section{Análisis de datos}

Para el análisis de datos se ha utilizado el programa estadístico SPSS (vs. 24). En todas las preguntas se calcularon diferentes estadísticos descriptivos, como medias, frecuencias y porcentajes.

\section{Resultados}

A continuación, se presentan los resultados obtenidos agrupados según los bloques en los que se ha estructurado el cuestionario.

\section{Datos personales}

Los resultados revelan que los participantes de la investigación eran en su mayoría bastante jóvenes, pues un 65\% aproximadamente tenía edades comprendidas entre los 20 y los 40 años (Tabla 1). De igual modo, es notorio que aproximadamente dos de cada tres docentes poseían 10 años o más de experiencia profesional (Tabla 2). En relación con los estudios cursados, el 78.1\% había obtenido el Grado o Diplomatura en Educación Infantil, siendo muy destacable que en torno al 30\% ostentaba otras diplomaturas o grados y un

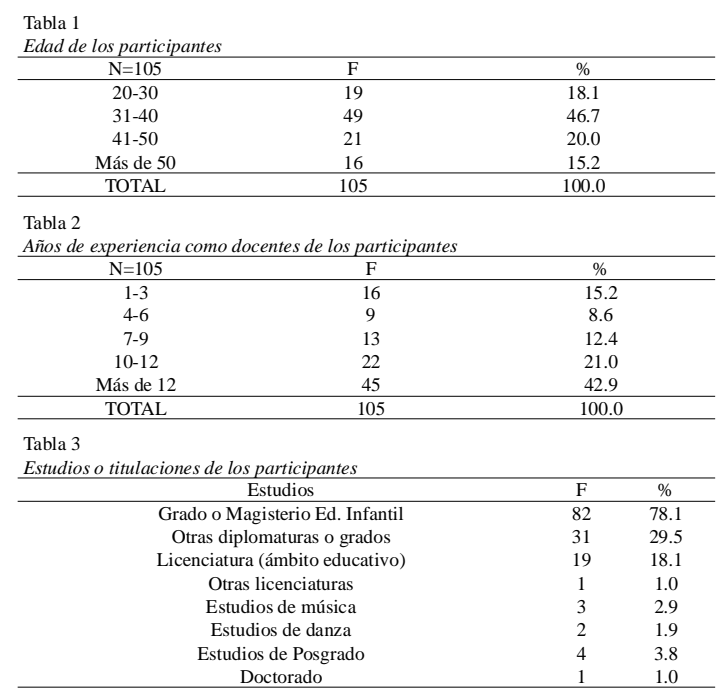


18\% una licenciatura relacionada con la educación. Por el contrario, solo un 5\% poseía estudios de danza o música (Tabla 3).

\section{Práctica en el aula}

En cuanto a los resultados consignados acerca de la práctica en el aula, la Tabla 4 indica que más del 93\% de los participantes se encontraba de acuerdo o totalmente de acuerdo con la inclusión de actividades de danza en sus programaciones docentes, frente al 6.7\% que manifestaba estar en desacuerdo o muy en desacuerdo. Asimismo, el 88.6\% de los docentes incluía este tipo de ejercicios con independencia de lo planificado (media= 3.13), frente a un $11.4 \%$ que no los incorporaba. Además, el 99\% de los docentes estaba de acuerdo o totalmente de acuerdo con la importancia de la práctica de la danza en el desarrollo integral del niño y consideraba que los contenidos de lenguaje corporal en el currículo de Educación Infantil son necesarios. Las medias de ambos ítems confirman que el rango de respuesta era muy cercano a 4 (totalmente de acuerdo).



En relación con la tipología de actividades de danza practicadas en el aula, el 81\% de los participantes realizaba canciones coreografiadas sencillas (Tabla 5). Al mismo tiempo, se aprecia que dos de cada tres docentes incorporaban ejercicios libres, espontáneos y de improvisación en sus actividades de aula. Igualmente, más del 72\% del profesorado practicaba poco o nada danzas creadas por ellos mismos. En cuanto a la aplicación de danzas populares, creadas por terceros o adaptaciones, aproximadamente la mitad de los docentes realizaba un uso poco frecuente de estas, frente a la otra mitad que las llevaba a la práctica en mayor medida.

\begin{tabular}{l} 
Tabla 5 \\
\begin{tabular}{ccccc} 
Tipología de actividades practicadas en el aula (porcentajes) & & \\
\hline Tipo de actividad & Nada & Poco & Mucho & Muchísimo \\
\hline Canciones coreografiadas sencillas & .0 & 19.0 & 62.9 & 18.1 \\
Ejercicios libres, espontáneos y de improvisación & 2.9 & 31.4 & 55.2 & 10.5 \\
Danzas creadas por mí & 8.6 & 63.8 & 21.0 & 6.6 \\
Danzas populares, creadas por terceros o & 1.9 & 46.7 & 44.8 & 6.6 \\
adaptaciones & & & &
\end{tabular} \\
\hline
\end{tabular}

\section{Formación del profesorado}

En lo referente a la formación de los docentes de Educación Infantil en danza, más del 95\% de los participantes consideraba que el docente de Educación Infantil debe tener una formación suficiente en esta disciplina (Tabla 6). Asimismo, dos tercios de la muestra reconocía que es adecuada su formación en danza para la etapa de Infantil, frente al tercio restante que manifestó encontrarse en desacuerdo o muy en desacuerdo con tal afirmación. Igualmente, dos de cada tres participantes conocían un repertorio amplio de ejercicios coreográficos y de danzas para Educación Infantil. En cuanto a cursos y seminarios de formación sobre esta temática, algo más del 77\% no consideraba suficiente la oferta existente, cuestión que confirma la media (2.02), situada plenamente en la categoría en desacuerdo. Paralelamente, en torno al 56\% expresaba haber participado en cursos de formación relacionados con la danza tras el término de sus estudios.

Tabla 6

Danza en Educación Infantil: formación del profesorado

\begin{tabular}{|c|c|c|c|c|c|c|}
\hline $\mathrm{N}=105$ & & $1 *$ & 2 & 3 & 4 & \\
\hline & F & $\%$ & $\mathrm{~F} \%$ & $\mathrm{~F} \%$ & $\mathrm{~F}$ & $\begin{array}{lll}\% & \text { M } & \text { DT } \\
\end{array}$ \\
\hline $\begin{array}{l}\text { El docente de Educación Infantil debe tener } \\
\text { formación suficiente en danza. }\end{array}$ & 0 & .0 & $\begin{array}{ll}5 & 4.8\end{array}$ & В 6158.1 & 139 & 37.13 .320 .56 \\
\hline $\begin{array}{l}\text { Mi formación en danza es adecuada para la etapa } \\
\text { en la que imparto clase. }\end{array}$ & 3 & 2.9 & & 45855.2 & 210 & 710.67 \\
\hline $\begin{array}{l}\text { Conozco un repertorio amplio de ejercicios } \\
\text { coreográficos y danzas para Educación Infantil. }\end{array}$ & 3 & 2.9 & & 46360.0 & 06 & 5.72 .690 .62 \\
\hline $\begin{array}{l}\text { Considero suficiente la oferta de cursos de } \\
\text { formación y seminarios relacionados con la danza. }\end{array}$ & 22 & 2 & & & 0 & $\begin{array}{ll}.0 & 2.020 .66 \\
\end{array}$ \\
\hline $\begin{array}{l}\text { He realizado cursos de formación relacionados con } \\
\text { la danza tras finalizar mis estudios. }\end{array}$ & & & & & & \\
\hline
\end{tabular}

\section{Actitud del alumnado}

En la Tabla 7 se observa que el 96\% de la muestra constataba que el alumnado participa de forma activa en ejercicios de danza. En la misma línea, el 100\% estaba de acuerdo o totalmente de acuerdo en que el alumnado se interesa por la práctica de dichas actividades. Además, para algo menos de la mitad de los docentes el comportamiento del alumnado se alteraba al realizar este tipo de ejercicios, frente al resto que no percibía dichas alteraciones.



\section{Disponibilidad de medios y recursos}

Por último, sobre la dedicación temporal destinada a la danza, el 62\% de los participantes señalaba disponer de tiempo necesario semanal para realizar estas actividades, aunque la media (2.63) de las respuestas consignadas la establecía entre los valores en desacuerdo o de acuerdo, con una desviación considerable (Tabla 8). El 83.8\% de los docentes consideró que debía aumentar el tiempo dedicado a este tipo de actividades en su práctica diaria. Con respecto al tiempo empleado para desarrollar contenidos de lenguaje corporal en relación con el resto de contenidos de la etapa, cerca del $43 \%$ indicó que era adecuado, mientras que el resto se mostró en desacuerdo o muy en desacuerdo con dicha afirmación. En este caso, la media (2.42) se ubicaba entre las categorías en desacuerdo o de acuerdo.

Tabla 8

Danza en Educación Infantil: dedicación temporal

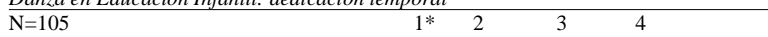
\begin{tabular}{llllllllllll} 
F & $\%$ & F & $\%$ & F & $\%$ & F & $\%$ & M & DT \\
\hline
\end{tabular} \begin{tabular}{llllllllllll}
\hline Dispongo de tiempo semanal necesario & 7 & 6.7 & 33 & 31.4 & 57 & 54.3 & 8 & 7.6 & 2.63 & 0.72
\end{tabular} para realizar actividades de danza.

Debo dedicar más tiempo a ejercicios de

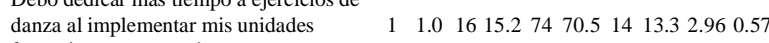
formativas programadas.

En relación con el resto de contenidos, el



adecuado.

adecuado. acuerdo

En lo referente a la disposición de un espacio para la realización de actividades de danza, aproximadamente la mi- 
tad de los docentes participantes afirmó disponer de un espacio apropiado para realizar ejercicios coreográficos y danzas, mientras que algo más de la mitad consideraba que no contaban con él (Tabla 9). Igualmente, más de la mitad de los docentes afirmó no tener un equipo de sonido adecuado.

Por último, con respecto a los recursos audiovisuales y bibliográficos, el 50\% del profesorado señaló que disponía de material audiovisual suficiente para la práctica de actividades de danza en el aula, porcentaje que disminuía al 39.1\% en el caso de la utilización de libros de texto (Tabla 9). Por el contrario, el 87.6\% de los participantes realizaba búsquedas a través de internet para este tipo de ejercicios y dos de cada tres creaban sus propias actividades coreográficas y danzas.

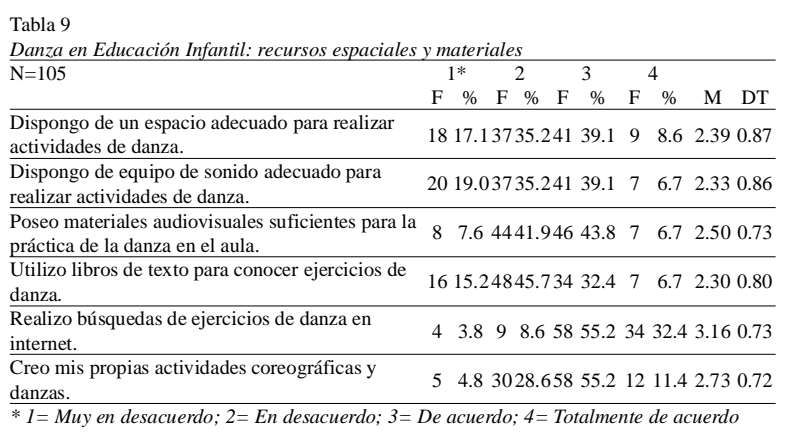

\section{Conclusiones}

Con respecto al grado de puesta en práctica de ejercicios coreográficos y danzas en el aula de Educación Infantil, se puede afirmar que la gran mayoría de los docentes participantes en el estudio planifica actividades de este tipo en sus programaciones e, incluso, las lleva a la práctica con independencia de las tareas programadas. Igualmente, enfatiza la importancia de los contenidos de lenguaje corporal del currículo, considerándolos de gran valor educativo y fundamentales para el desarrollo integral del niño, hecho que reafirma las palabras de Pedrero (2013) y Ríos (2017).

Asimismo, los docentes de Educación Infantil conocen un amplio repertorio de actividades de danza y, aunque piensan que su formación en estas disciplinas es suficiente, consideran necesario seguir formándose. Al respecto, opinan que la oferta de cursos de formación y seminarios es escasa. Estos resultados continúan la línea de los estudios de Troya \& Cuéllar (2013), que constatan la escasa formación del profesorado en danza. Debe recordarse que solo un 5\% posee estudios de danza o música, si bien más de la mitad del profesorado había participado en algún curso relacionado con la danza tras la finalización de sus estudios universitarios.

En cuanto a la opinión del profesorado sobre la actitud del alumnado de Educación Infantil durante la praxis de la danza, los docentes destacan mayoritariamente que el alumnado participa de manera activa en este tipo de ejercicios, siendo la totalidad los que evidencian interés ante los mismos. Coincidiendo con el estudio de Llorens \& Díaz (2018), el porcentaje del profesorado que señala alteraciones del ritmo normal de clase durante estas actividades es elevado, sin embargo, no puede afirmarse que sea un condicionante para su puesta en práctica, pues más del 90\% de la muestra incluía la práctica de la danza en sus aulas.
Dos tercios de los docentes de Infantil dispone de tiempo semanal suficiente para trabajar actividades de danza, aunque para un poco más de la mitad es insuficiente el tiempo empleado a desarrollar los contenidos de lenguaje corporal programados. Es destacable que una mayoría bastante amplia (83.8\%) considera que debe incrementar su dedicación al trabajo de la misma en sus aulas. Al respecto, debe recordarse que la Orden ECI/3960/2007 de 19 de diciembre, por la que se establece el currículo y se regula la ordenación de la Educación Infantil, especifica de forma clara que la organización temporal debe orientarse para que exista tiempo para todo y para todos. En futuros estudios, sería interesante indagar en los motivos que hacen que el profesorado no dedique más tiempo del que desea a esta disciplina.

Por último y en cuanto a recursos materiales, sorprende que la mitad de los docentes no disponga de un espacio idóneo para llevar a cabo ejercicios de danza, no tengan un equipo de sonido que cumpla unos requisitos mínimos y no dispongan de material audiovisual para trabajar estos contenidos. La falta de recursos bibliográficos y audiovisuales es compensada en su gran mayoría por búsquedas realizadas en internet y la creación de producciones corporales propias.

Una vez constatada la presencia de la danza en la etapa de Educación Infantil, sería el momento de emprender otros estudios para analizar los recursos existentes, así como del repertorio o la metodología de ejercicios utilizados por algunos docentes, con el fin de conocer más a fondo la realidad del aula. Asimismo, sería necesario ahondar en las causas que generan una escasa oferta formativa o la insuficiencia de medios y recursos en relación con esta temática.

\section{Referencias}

Águila, C., \& López, J. J. (2019). Cuerpo, corporeidad y educación: una mirada reflexiva desde la Educación Física. Retos. Nuevas tendencias en Educación Física, Deporte y Recreación, 35, 413-421. Recuperado de https:/ /recyt.fecyt.es/index.php/retos/article/view/62035

Bernal, A. M. (2016). La importancia de la danza inclusiva en la formación docente. Revista Horizontes Pedagógicos, 18(2), 134-148. Recuperado de https:// revistas.iberoamericana.edu.co/index.php/ rhpedagogicos/article/view/1147

Bisquerra, R. (Coord.) (2009). Metodología de la Investigación Educativa. Madrid: La Muralla.

Cañal, F., \& Cañal, C. (2001). Música, Danza y Expresión Corporal en educación infantil y primaria. Tomo 1. Sevilla: Junta de Andalucía, Consejería de Educación y Ciencia. Recuperado de https:// www.observatoriodelainfancia.es/ficherosoia/documentos/1155_d_69805bc7f_musicadanza.pdf

Carrascosa, E. (2015). Entrevista a Verena Maschat. Eufonía: Didáctica de la música, 64, 1-8. Recuperado de https://docplayer.es/25989386-Entrevista-a-verenamaschat.html

Cuéllar. M. J., \& Pestano, M. (2013). Formación del Profesorado en Expresión Corporal: planes de estudio y Educación Física. Retos. Nuevas tendencias en Educación Física, Deporte y Recreación, 24, 123-128. Recuperado 
de https://recyt.fecyt.es/index.php/retos/article/view/ 34542

Díaz, A. M. (2018). Adquisición de conceptos musicales a través de la danza. DEDiCA. Revista de Educação e Humanidades, 13, 109-119. Recuperado de http:// revistaseug.ugr.es/index.php/dedica/article/view/7243/ 6440

Fernández, B., \& Arias, J. R. (2013). La Expresión Corporal como fuente de aprendizaje de nociones matemáticas espaciales en Educación Infantil. Retos. Nuevas tendencias en Educación Física, Deporte y Recreación, 24, 158-164. Recuperado de https://dialnet.unirioja.es/descarga/articulo/4482750.pdf

Ferreira, M. A. (2009). Un enfoque pedagógico de la danza. Educación Física Chile, 268, 9-21. Recuperado de https:/dialnet.unirioja.es/descarga/articulo/3237201.pdf

Herranz, A., \& López, V. M. (2015). La expresión corporal en educación infantil. La Peonza: Revista de Educación Física para la paz, 10, 23-43. Recuperado de https:// dialnet.unirioja.es/descarga/articulo/5367747.pdf

Lení, V., \& Wey, W. (2015). Movimiento y expresión corporal en Educación Infantil. Madrid: Narcea Ediciones.

Llorens, J. B. (2016). La diversidad en el aula de infantil a través de la danza integrada. Revista Interuniversitaria de Investigación sobre Discapacidad e Interculturalidad, 10(1), 55-56. Recuperado de http:// www.grupo-edi.com/anuario_list.php?ano=2016(1)

Llorens, J. B., \& Díaz, A. M. (2018). Aspectos corporais e sociais a serem considerados numa educação inclusiva. Movimento (ESEFID/UFRGS), 24(4), 1219-1234. Recuperado de https://seer.ufrgs.br/Movimento/article/ download/82614/51838
Martín, M. J. (2005). Del movimiento a la danza en la educación musical. Educatio Siglo XXI, 23, 125-139. Recuperado de http://hdl.handle.net/10201/26748

Orden ECI/3960/2007, de 19 de diciembre, por la que se establece el currículo y se regula la ordenación de la educación infantil. Boletín Oficial del Estado, $n^{\circ}$ 5, 2008, 5 enero. Recuperado de https://www.boe.es/boe/dias/2008/ 01/05/pdfs/A01016-01036.pdf

Pedrero, C. (2013). Danza en Educación Primaria. Educatio Siglo XXI, 31(1), 129-148. Recuperado de http:// revistas.um.es/educatio/article/view/175101/148241

Pérez-Aldeguer, S. (2012). Didáctica de la Expresión Musical en Educación Infantil. Valencia: Psylicom.

Real Decreto 1630/2006, de 29 de diciembre, por el que se establecen las enseñanzas mínimas del segundo ciclo de Educación infantil. Boletín Oficial del Estado, $n^{\circ}$ 4, 2007, 4 enero. Recuperado de https://www.boe.es/boe/dias/ 2007/01/04/pdfs/A00474-00482.pdf

Ríos, P. (2017). El movimiento y la danza en la educación musical. En R. Cremades (Coord.), Didáctica de la Educación Musical en Primaria (p. 99-128). Madrid: Ediciones Paraninfo.

Troya, Y., \& Cuéllar, M. J. (2013). Formación docente y tratamiento de la danza en Canarias: evaluación desde la Educación Física. Retos. Nuevas tendencias en Educación Física, Deporte y Recreación, 24, 165-170. Recuperado de https://www.redalyc.org/pdf/3457/345732290033.pdf

Vicente, G., Ureña, N., Gómez, M., \& Carrillo, J. (2010). La danza en el ámbito educativo. Retos. Nuevas tendencias en Educación Física, Deporte y Recreación, 17, 42-45. Recuperado de https://www.redalyc.org/ pdf/3457/345732283009.pdf
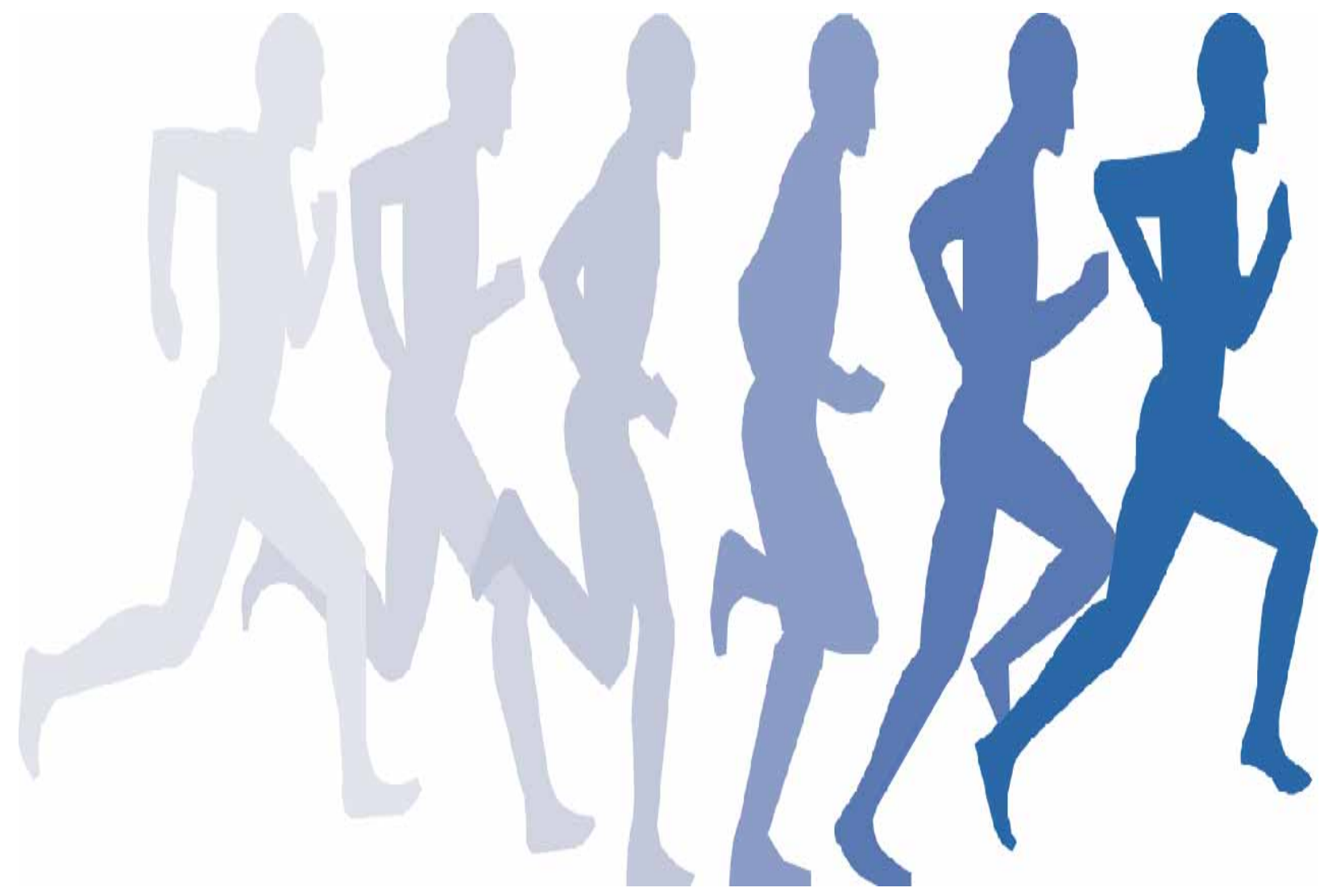